\title{
Modeling and non-linear self-tuning robust trajectory control of an autonomous underwater vehicle
}

\author{
THOR I. FOSSEN $\dagger$ and JENS G. BALCHEN $\dagger$
}

Keywords: Non-linear identification, non-linear control, robust control, self-tuning, autonomous underwater vehicle

\begin{abstract}
A non-linear self-tuning algorithm is demonstrated for an autonomous underwater vehicle. Tighter control is achieved by a non-linear parameter identification algorithm which reduces the parameter uncertainty bounds. Expensive hydrodynamic tests for parameter determination can thus be avoided. Excellent tracking performance and robustness to parameter uncertainty are guaranteed through a robust control strategy based on the estimated parameters. The nonlinear control law is highly robust for imprecise models and the neglected dynamics. The non-linear self-tuning control strategy is simulated for the horizontal positioning of an underwater vehicle.
\end{abstract}

\section{Introduction}

Conventional multi-variable and adaptive controllers are difficult to design for autonomous unmanned underwater vehicles. This is because their strongly coupled dynamics are highly non-linear and vary according to a vessel's operating point. An alternative design procedure is self-tuning non-linear control strategy based on Robust Trajectory Control (RTC), Asada and Slotine (1986). The non-linear control law which is calculated from a simplified model of the vehicle shows good performance and robustness. The main advantage of this control strategy is that there is one controller for each degree of freedom and only a restricted number of parameters to identify.

The RTC algorithm combined with a suitable non-linear recursive identification algorithm is easy to implement. An adequate non-linear identification algorithm such as the Non-linear Recursive Prediction Error Method (NRPE) (Zhou (1987)) is well suited for this purpose. An approximate model can be identified off-line by a self-test executed by the vehicle. If the vehicle undergoes a configuration change, the self-test is executed autonomously under computer control. Before the mission, the vehicle moves in a fixed pattern given by a cycle of step inputs to each thruster in turn. During the run the position data is logged. This data is used as input for the identification algorithm. The self-test can be programmed to be executed by the computer on the vessel or as a previous off-line calculation. The self-tuning algorithm does not need any $a$ priori information of the vehicle's hydrodynamic parameters. As a result of this, it is possible to schedule the control gains without performing expensive model tests each time.

The non-linear identification algorithm will be tested on a SPRINT 101 underwater vehicle in the Ocean Basin at the Norwegian Marine Technology

Received 15 September 1988

$\dagger$ Center for Robotic Research, Division of Engineering Cybernetics, The Norwegian Institute of Technology, N7034 Trondheim, Norway. 
Research Institute. Later, several tests will be performed as a part of the Norwegian Robotic Research Program ${ }^{1}$, Norwegian Institute of Technology.

\section{Modeling of an autonomous underwater vehicle}

The dynamic behaviour of an underwater vehicle can be described by Newton's laws of linear and angular momentum formulated in a vessel-based coordinate system. The equations of motion for an underwater vehicle in 6DOF may be written in the same compact state space form as robot manipulators (Fossen (1987)) where

$$
\begin{aligned}
M \ddot{\boldsymbol{q}} & =N(\dot{\boldsymbol{q}}, \boldsymbol{x})+B \boldsymbol{U} \\
\dot{\boldsymbol{x}} & =J(\boldsymbol{x}) \dot{\boldsymbol{q}}
\end{aligned}
$$

The bold type indicates vectors while the dot is the derivative with respect of time. According to the SNAME notation (Society of Naval Architects and Marine Engineers (1952)) the vessel's velocity vector is considered to be $\dot{\boldsymbol{q}}=[u, v, w, p, q, r]^{T}$ and the earth-fixed position/orientation vector is $\boldsymbol{x}=[x, y, z, \phi, \theta, \psi]^{T}$.

$M$ is 6 by 6 inertia matrix including the vehicle's mass, added mass and inertia tensor.

$\boldsymbol{M}=\left[\begin{array}{cccccc}m-X_{\dot{u}} & -X_{\dot{v}} & -X_{\dot{w}} & -X_{\dot{p}} & m z_{g}-X_{\dot{q}} & -m y_{g}-X_{\dot{r}} \\ -X_{\dot{v}} & m-Y_{\dot{v}} & -Y_{\dot{w}} & -m z_{g}-Y_{\dot{p}} & -Y_{\dot{q}} & m x_{g}-Y_{\dot{r}} \\ -X_{\dot{w}} & -Y_{\dot{w}} & m-Z_{\dot{w}} & m y_{g}-Z_{\dot{p}} & -m x_{g}-Z_{\dot{q}} & -Z_{\dot{r}} \\ -X_{\dot{p}} & -m z_{g}-Y_{\dot{p}} & m y_{g}-Z_{\dot{p}} & I x-K_{\dot{p}} & -I x y-K_{\dot{q}} & -I z x-K_{\dot{r}} \\ m z_{g}-X_{\dot{q}} & -Y_{\dot{q}} & -m x_{g}-Z_{\dot{q}} & -I x y-K_{\dot{q}} & I y-M_{\dot{q}} & -I y z-M_{\dot{r}} \\ -m y_{g}-X_{\dot{r}} & m x_{\dot{g}}-Y_{\dot{r}} & -Z_{\dot{r}} & -I z x-K_{\dot{r}} & -I y z-M_{\dot{r}} & I z-N_{\dot{r}}\end{array}\right]$

$m$ is the vehicle's mass including water in free floating spaces, $I_{i j}$ is the product of inertia about the axes $\mathrm{i}$ and $\mathrm{j}$ and $\left(x_{g}, y_{g}, z_{g}\right)$ is the center of gravity. The added mass is represented in terms of slow motion derivatives. As an example $\boldsymbol{Y}_{\dot{w}} \cdot \dot{w}$ is the force in $y$-direction caused by an acceleration in the z-direction, where $Y_{\dot{w}}=\partial Y / \partial \dot{w}$. Newman (1977) has shown that for a rigid body moving in an ideal fluid the mass matrix is symmetrical i.e. $M=M^{T}$. In a real fluid these 36 elements may all be distinct. Experience has shown that the numerical values of added mass in a real fluid are usually in good agreement with those obtained from ideal theory (Wendel (1956)).

The $J$ matrix represents the coordinate transformation matrix between the earth- and vessel-based coordinate systems. The coordinate transformation is related through the functions of the Euler angles: roll $(\phi)$, pitch $(\theta)$ and yaw $(\psi)$.

$$
J(x)=\left[\begin{array}{cc}
J_{1} & 0 \\
0 & J_{2}
\end{array}\right]
$$

where $J_{1}$ represents the linear velocity transformation and $J_{2}$ is the angular velocity transformation.

${ }^{1}$ The Norwegian Robotic Research Program is sponsored by the Royal Norwegian Council for Scientific and Industrial Research and will include $\mathbf{4 0}$ doctoral candidates in the general field of robotics 


$$
\begin{aligned}
J_{1} & =\left[\begin{array}{ccc}
c(\psi) c(\theta) & -s(\psi) c(\phi)+c(\psi) s(\theta) s(\phi) & s(\psi) s(\phi)+c(\psi) c(\phi) s(\theta) \\
s(\psi) c(\theta) & c(\psi) c(\phi)+s(\phi) s(\theta) s(\psi) & -c(\psi) s(\phi)+s(\theta) s(\psi) c(\phi) \\
-s(\theta) & c(\theta) s(\phi) & c(\theta) c(\phi)
\end{array}\right] \\
J_{2} & =\left[\begin{array}{ccc}
1 & s(\phi) t(\theta) & c(\phi) t(\theta) \\
0 & c(\phi) & -s(\phi) \\
0 & s(\phi) / c(\theta) & c(\phi) / c(\theta)
\end{array}\right]
\end{aligned}
$$

where $c(\cdot)=\cos (\cdot), s(\cdot)=\sin (\cdot)$ and $t(\cdot)=\tan (\cdot)$. Because of the symmetry properties we have $J_{1}^{-1}=J_{1}^{T}$. Notice $J_{2}^{-1} \neq J_{2}^{T}$.

$\boldsymbol{N}$ is a non-linear vessel-based vector term including hydrodynamic, rigid-body kinematic and hydrostatic forces. The non-linear vector $N$ is written

$$
N(\dot{q}, x)=N_{h}(\dot{q})+N_{g}(x)
$$

where the transformed gravity vector is

$$
N_{g}(x)=\left[\begin{array}{l}
-(W-B) \sin \theta \\
(W-B) \cos \theta \sin \phi \\
(W-B) \cos \theta \cos \phi \\
\left(y_{g} W-y_{B} B\right) \cos \theta \cos \phi-\left(z_{g} W-z_{B} B\right) \cos \theta \sin \phi \\
-\left(x_{g} W-x_{B} B\right) \cos \theta \cos \phi-\left(z_{g} W-z_{B} B\right) \sin \theta \\
\left(x_{g} W-x_{B} B\right) \cos \theta \sin \phi+\left(y_{g} W-y_{B} B\right) \sin \theta
\end{array}\right]
$$

$\left(x_{g}, y_{g}, z_{g}\right)$ is the center of gravity, $\left(x_{b}, y_{b}, z_{b}\right)$ is the center of buoyancy and $W$ and $B$ are the vehicle's gravity and buoyancy forces respectively.

The modeling problem is to determine the $N_{h}$ vector and the added mass coefficients in the mass matrix. The $N$ vector may be modelled as a Taylor series expansion of $\dot{\boldsymbol{q}}=[u, v, w, p, q, r]^{T}$ where small terms are neglected. Another way is to perform simulation studies of the actual vehicle on a computer. These simulation results should be compared to model or full-scale tests to fit the coefficients to a selected model. For parameter identification it is recommended to reduce the number of parameters as much as possible. Often this will improve the identifiability. Hydrodynamic coefficients which are known with sufficient accuracy may be included in the model as constants with no need for parameter estimation.

$B$ is the control matrix which depends on the vehicle's thruster configuration while $\boldsymbol{U}$ is the control vector.

\section{Non-linear self-tuning control strategy}

The self-tuning control strategy is based on off-line non-linear parameter identification. If an estimation of the Kalman filter gains for the state update are included, these may be executed as an on-line calculation, due to the time-varying conditions for underwater disturbances and changes in operating conditions, Fig. 1.

\subsection{Non-linear recursive prediction error method}

The Non-linear Recursive Prediction Error method (NRPE) (Zhou (1987)) is an extension from the Recursive Prediction Error method (RPE) (Ljung (1987)). Both methods are based on a parameter update through a Gauss-Newton search direction. The only difference between the linear and non-linear cases is the calculation of 


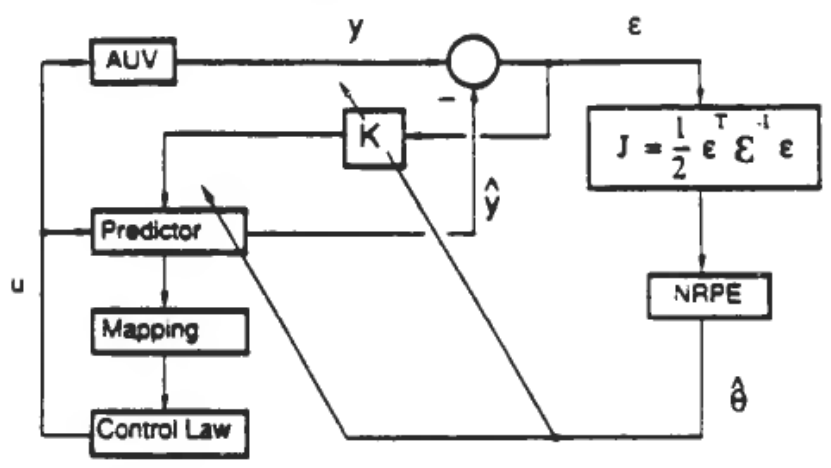

Figure 1. Self-tuning control scheme.

the prediction output and the gradient. In the non-linear case they are based on an approximate second order non-linear filter combined with Ljung's innovations filter. This non-linear prediction filter model includes second order correction terms to avoid biased estimates. The estimates are unbiased for non-linearities of the order of less than two. The method has been developed both in discrete and continuousdiscrete versions by Zhou (1987).

Based on the ideas of Ljung's innovations filter model where the Kalman filter gain $K(\theta)$ may be treated as an element of the parameter vector $\theta$ to be estimated, the non-linear continuous-discrete predictor with linear measurement is written

$$
\begin{aligned}
\frac{d \hat{x}\left(t \mid t_{i}^{+}, \theta\right)}{d t} & =f\left(\theta, u ; t, \hat{x}\left(t \mid t_{i}^{+}, \theta\right)+B_{x}\left(t \mid t_{i}, \theta\right)\right. \\
\varepsilon\left(t_{i+1}\right) & =y\left(t_{i+1}\right)-D(\theta) \hat{x}\left(t_{i+1}^{-}, \theta\right) \\
\hat{x}\left(t_{i+1}^{+}, \theta\right) & =\hat{x}\left(t_{i+1}^{-}, \theta\right)+K\left(t_{i+1}, \theta\right) \varepsilon\left(t_{i+1}\right)
\end{aligned}
$$

where $\boldsymbol{x}$ is the state vector, $\boldsymbol{y}$ the measurement vector, $f(\cdot)$ the non-linear vector function and $D$ the measurement matrix.

The $k^{\prime}$ th component of the second order bias correction $\boldsymbol{B}_{\boldsymbol{x}}$ is given by

$$
B_{x k}=\frac{1}{2} \operatorname{tr}\left\{\frac{\partial^{2} f_{k}(\hat{x}, u, t)}{\partial \hat{x}^{2}} X(t)\right\}
$$

The covariance matrix $\boldsymbol{X}$ and the gradient matrix $\Xi$ are calculated through the equations

$$
\begin{aligned}
\Phi\left(t_{i}\right) & =\left.\frac{\partial f\left(\theta, u ; t, \hat{x}\left(t \mid t_{i}, \theta\right)\right)}{\partial x}\right|_{\theta=\hat{\theta}\left(t_{i}\right)} \\
\frac{d X\left(t \mid t_{i}^{+}\right)}{d t} & =\Phi\left(t_{i}\right) X\left(t \mid t_{i}^{+}\right)+X\left(t \mid t_{i}^{+}\right) \Phi^{T}\left(t_{i}\right)+V\left(t_{i}\right) \\
R^{*}\left(t \mid t_{i}^{+}, \theta\right) & =\frac{\partial}{\partial \theta}\left\{\left.f\left(\theta, u ; t, \hat{x}\left(t \mid t_{i}^{+}, \theta\right)+B_{x}\left(t \mid t_{i}^{+}, \theta\right)\right\}\right|_{\theta=\hat{\theta}\left(t_{i}\right)}\right. \\
S^{*}\left(t_{i}\right) & =\left.\frac{\partial}{\partial \theta}\left\{f(\theta, u ; t, \hat{x})+B_{x}\left(t \mid t_{i}^{+}, \theta\right)\right\}\right|_{\theta=\hat{\mathbf{\theta}}\left(t_{i}\right)} \\
\frac{d \Xi\left(t \mid t_{i}^{+}, \theta\right)}{d t} & =R^{*}\left(t \mid t_{i}^{+}, \theta\right)+S^{*}\left(t_{i}\right)
\end{aligned}
$$


where $V$ is the noise covariance matrix of the process. After the integration of the above equations, $\hat{x}\left(t_{i+1}^{-}\right), X\left(t_{i+1}^{-}\right)$and $\Xi\left(t_{i+1}^{-}\right)$are available. The recursive scheme is then written

$$
\begin{aligned}
D_{\theta}\left(t_{i}\right) & =\left.\frac{\partial}{\partial \theta}[D(\theta) \hat{x}]\right|_{\theta=\hat{\theta}\left(t_{i}\right)} \\
\Psi^{T}\left(t_{i+1}\right) & =D \Xi\left(t_{i+1}^{-}, \theta\right)+D_{\theta}\left(t_{i}\right) \\
y\left(t_{i+1}\right) & =D \hat{x}\left(t_{i+1}^{-}\right) \\
\varepsilon\left(t_{i+1}\right) & =y\left(t_{i+1}\right)-\hat{y}\left(t_{i+1}\right) \\
\hat{\varepsilon}\left(t_{i+1}\right) & =\hat{\varepsilon}\left(t_{i}\right)+\alpha\left(t_{i+1}\right)\left[\varepsilon\left(t_{i+1}\right) \varepsilon^{T}\left(t_{i+1}\right)-\hat{\varepsilon}\left(t_{i}\right)\right] \\
\Gamma\left(t_{i+1}\right) & =\Gamma\left(t_{i}\right)+\alpha\left(t_{i+1}\right)\left[\Psi\left(t_{i+1}\right) \hat{\varepsilon}^{-1}\left(t_{i+1}\right) \Psi^{T}\left(t_{i+1}\right)-\Gamma\left(t_{i}\right)\right] \\
\hat{\theta}\left(t_{i+1}\right) & =\hat{\theta}\left(t_{i}\right)+\alpha\left(t_{i+1}\right) \Gamma^{-1}\left(t_{i+1}\right) \Psi\left(t_{i+1}\right) \hat{\mathcal{E}}^{-1}\left(t_{i+1}\right) \varepsilon\left(t_{i+1}\right) \\
\hat{\boldsymbol{x}}\left(t_{i+1}^{+}, \theta\right) & =\hat{x}\left(t_{i+1}^{-}, \theta\right)+K\left(t_{i+1}, \theta\right) \varepsilon\left(t_{i+1}\right) \\
X\left(t_{i+1}^{+}\right) & =\left[I-K\left(t_{i+1}, \theta\right) D\right] X\left(t_{i+1}^{-}\right) \\
K_{\theta}\left(t_{i+1}, \theta\right) & =\left.\left[\frac{\partial}{\partial \theta} K\left(t_{i+1}, \theta\right)\right]\right|_{\theta=\hat{\theta}\left(t_{i}\right)} \varepsilon\left(t_{i+1}\right) \\
\Xi\left(t_{i+1}^{+}, \theta\right) & =\left[I-K\left(t_{i+1}, \theta\right) D\right] \Xi\left(t_{i+1}^{-}, \theta\right)+K_{\theta}\left(t_{i+1}, \theta\right)-K\left(t_{i+1}, \theta\right) D_{\theta}\left(t_{i}\right)
\end{aligned}
$$

where $\Gamma$ is the Hessian, the second derivative of the performance index $J(\theta)=$ $\frac{1}{2} \varepsilon^{T}(t) \varepsilon^{-1}(t) \varepsilon(t)$ with respect to $\theta, \Psi\left(t_{i}, \theta\right)$ is the gradient of the predicted output $\hat{\boldsymbol{y}}\left(t_{i} \mid \boldsymbol{\theta}\right)$ with respect to $\theta, \varepsilon(t)$ is the covariance matrix of the prediction error and $\alpha\left(t_{i}\right)$ is the step size factor. The step size factor is suggested as

$$
\alpha\left(t_{i}\right)=\frac{1}{i+1}
$$

In the case where all states are measured, the matrix $X\left(t_{i}\right)$ may be replaced by $\hat{\varepsilon}\left(t_{i}\right)$ and Eqns 12, 13 and 25 removed. The use of the discrete version of the NRPE is discussed in Zhou (1987).

\subsection{Non-linear robust trajectory control}

The non-linear robust trajectory methodology guarantees tracking precision. Robustness to the uncertainty is explicitly guaranteed even for quite simplified models. The methodology is based on a single-input single-output (SISO) formulation of the control problem. One single controller is designed for each degree of freedom rather than a high order complex multivariable controller. The control methodology accounts for the multivariable effects and cross-coupling terms in an easy way without any stability problems. The following equations are based on a second order system (Yoerger and Slotine (1985)) described by

$$
\ddot{x}=f(X, \theta ; t)+b(X, \theta ; t) U
$$

The parameter vector $\theta$ is calculated from the NRPE method, $f(\cdot)$ is the systems time-varying dynamics, $b(\cdot)$ is the control gain, $X=[x, \dot{x}]^{T}$ is the state vector, $U$ is the control force and $x$ is the actual state. 
The uncertainty of the nonlinear function $f(\cdot)$ and the control gain $b(\cdot)$ is known to within the following margins

$$
|\hat{f}(\boldsymbol{X}, \boldsymbol{\theta} ; t)-f(\boldsymbol{X} ; t)| \leqslant F(\boldsymbol{X}, \boldsymbol{\theta} ; t)
$$

where $\hat{f}(\boldsymbol{X}, \boldsymbol{\theta} ; t)$ is the estimate of $f(X ; t)$ and

$$
\beta^{-1} \leqslant \frac{\hat{b}(\boldsymbol{X}, \boldsymbol{\theta} ; t)}{b(X ; t)} \leqslant \beta
$$

where $\hat{b}(X, \theta ; t)$ is the estimate of $b(X ; t)$ and $\beta$ is the gain margin of the control system. A sliding surface is defined as

$$
s(X ; t)=\dot{\tilde{x}}+\lambda \tilde{x}, \quad \lambda>0
$$

where $\tilde{X}=[\tilde{x}, \dot{\tilde{x}}]^{T}$ is the tracking error vector and $\tilde{x}=x-x_{d}$.

This corresponds to a time-varying surface in the state space $R^{2}$, that is a line moving with the point $\left(x_{d}, \dot{x}_{d}\right)$ with slope, $\lambda$, Fig. 2 , where $\lambda$ is a design parameter interpreted as the desired control bandwidth. Perfect tracking is defined as remaining along the surface $s(X, t)=0$. In order to have perfect tracking $x(t)=x_{d}(t)$ the sliding condition

$$
\frac{1}{2} \frac{d\left(s^{2}\right)}{d t} \leqslant-\eta|s|
$$

has to be satisfied where $\eta$ is a small positive constant. The sliding condition directly follows from Lyapunov's 2nd method where $V(s)=\frac{1}{2} s^{2}$ is selected as the Lyaponov function. A control law that satisfies this condition (Asada and Slotine (1986)) is

$$
\begin{aligned}
U & =\hat{b}^{-1}(\boldsymbol{X}, \boldsymbol{\theta} ; t)[\tilde{U}-\tilde{K}(\boldsymbol{X}, \boldsymbol{\theta} ; t) \text { sat }(s / \phi)] \\
\tilde{U} & =-\hat{f}(\boldsymbol{X}, \boldsymbol{\theta} ; t)+\ddot{x}_{d}-\lambda \dot{\tilde{x}}
\end{aligned}
$$

where $\tilde{K}(X, \theta ; t)$ is a discontinuous term across the sliding surface satisfying Eqn 33 and sat $(\cdot)$ is the saturation function. Adding this discontinuous term to the control law guarantees robust stability within the specified uncertainty bounds.

$$
\begin{array}{ll}
\text { sat }(x)=\operatorname{sgn}(x) & |x| \geqslant 1 \\
\text { sat }(x)=x & \text { otherwise }
\end{array}
$$

and

$$
\begin{array}{ll}
\operatorname{sgn}(x)=+1 & x>0 \\
\operatorname{sgn}(x)=-1 & x \leqslant 0
\end{array}
$$

To avoid chattering, the control law is smoothed out in a thin boundary layer $\phi$ around the sliding surface, Fig. 2 . In this case the sliding condition is modified to

$$
\frac{1}{2} \frac{d\left(s^{2}\right)}{d t} \leqslant(\dot{\phi}-\eta)|s|
$$

The desired thickness of the boundary layer is calculated from

$$
\begin{aligned}
& \dot{\phi}(t)=-\lambda \phi(t)+\beta K\left(X_{d}, \theta ; t\right) \text { if } K\left(X_{d}, \theta ; t\right) \geqslant \frac{\lambda \phi(t)}{\beta} \\
& \dot{\phi}(t)=-\frac{\lambda \phi(t)}{\beta^{2}}+K\left(X_{d}, \theta ; t\right) / \beta \quad \text { if } \quad K\left(X_{d}, \theta ; t\right)<\frac{\lambda \phi(t)}{\beta}
\end{aligned}
$$



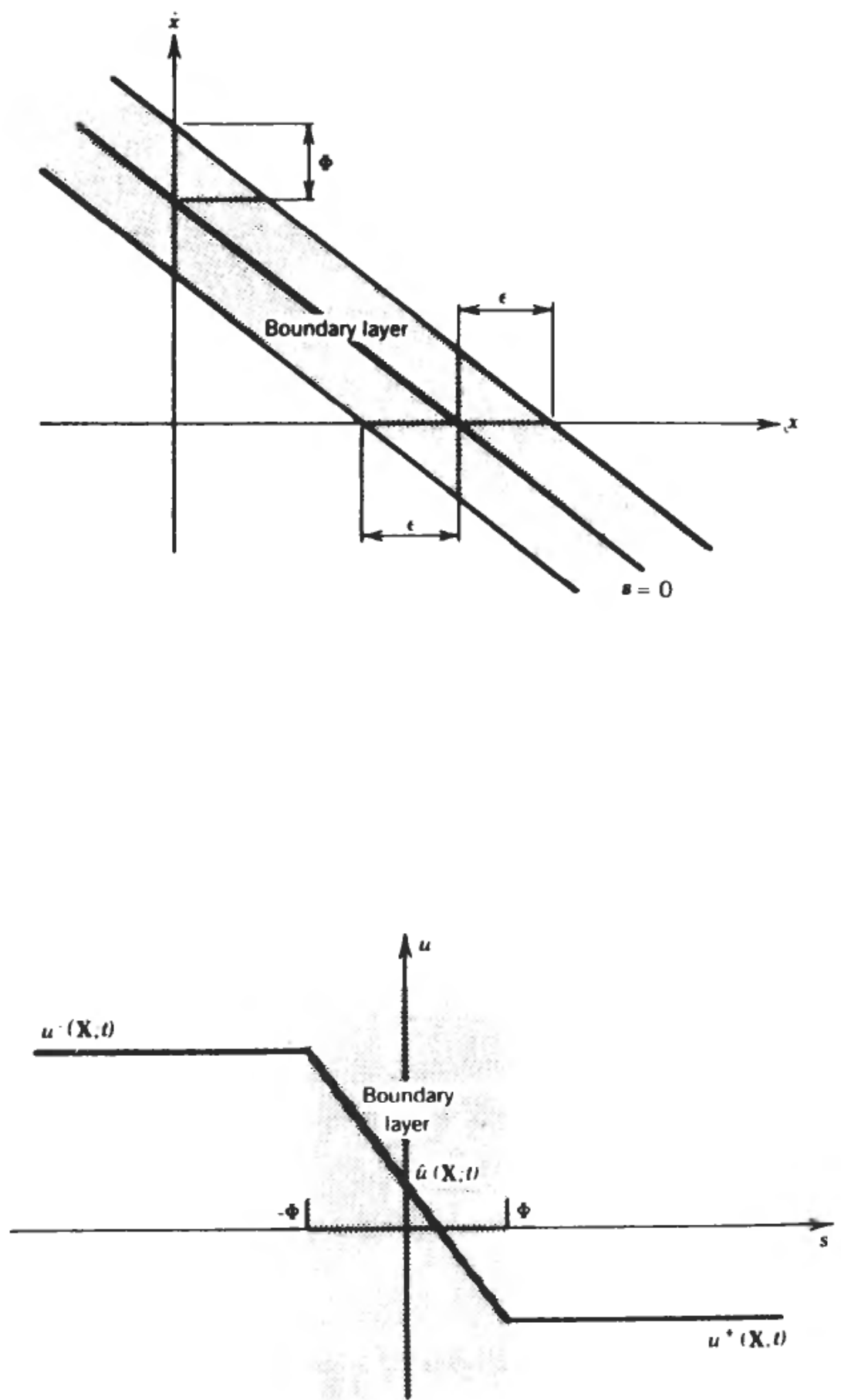

Figure 2. Sliding surface and the boundary layer (Asada and Slotine (1986)). 
with the initial condition

$$
\phi(0)=\beta K\left(\mathbf{X}_{d}(0), \boldsymbol{\theta} ; 0\right) / \lambda
$$

$\tilde{K}(X, \theta ; t)$ is calculated from

$$
\tilde{K}(\boldsymbol{X}, \boldsymbol{\theta} ; t)=K(\boldsymbol{X}, \boldsymbol{\theta} ; t)-K\left(\boldsymbol{X}_{\mathrm{d}}, \boldsymbol{\theta} ; t\right)+\lambda \phi(t) / \boldsymbol{\beta}
$$

where

$$
K(X, \theta ; t)=F(X, \theta ; t)+\eta
$$

The use of the discrete version of robust trajectory control is discussed in Delonga, Slotine and Yoerger (1987).

\section{Application to a continuous system}

\subsection{Simulation study}

A simulation study was performed to demonstrate the non-linear parameter identification and the control strategy. A 3DOF model for the simulation of an underwater vehicle's horizontal motion was chosen as

$$
M \ddot{q}=N(\ddot{q})+U
$$

where

$$
\begin{aligned}
\dot{\boldsymbol{q}} & =[u, v, r]^{T} \\
\boldsymbol{U} & =\left[U_{x}, U_{y}, U_{r}\right]^{T}
\end{aligned}
$$

and

$$
\boldsymbol{M}=\left[\begin{array}{rrr}
143 & 3 & 8 \\
3 & 163 & 5 \\
8 & 5 & 99
\end{array}\right]
$$

An adequate non-linear vector $N$ is

$$
N(\dot{q})=\left[\begin{array}{l}
-120 u|u| \\
-150 v|v|-30 u|v|-10 v|u|-50 r|r| \\
-200 r|r|-10 v|r|
\end{array}\right]
$$

A simplified non-linear model with 6 unknown parameters for identification was used to demonstrate the self-tuning non-linear control algorithm. The predictor does not include any off-diagonal elements in the mass matrix and is simply written as

$$
\begin{aligned}
\dot{\hat{u}} & =X_{u|| v \mid} \hat{u}|\hat{u}|+X_{t} U_{x} \\
\dot{\hat{v}} & =Y_{v|v|} \hat{v}|\hat{v}|+Y_{t} U_{y} \\
\dot{\hat{r}} & =N_{r|r|} \hat{r}|\hat{r}|+N_{t} U_{r}
\end{aligned}
$$

The non-linear functions $\hat{f}_{k}$ and the control gains $\hat{b}_{k}$ for the Robust Trajectory Controller are recognized as

$$
\begin{array}{ll}
\hat{f}_{1}=X_{u|v|} \hat{u}|\hat{u}|, & \hat{b}_{1}=X_{t} \\
\hat{f}_{2}=Y_{v|v|} \hat{v}|\hat{v}|, & \hat{b}_{2}=Y_{t} \\
\hat{f}_{6}=N_{r|v|} \hat{r}|\hat{r}|, & \hat{b}_{6}=N_{t}
\end{array}
$$




\subsection{Simulation result}

The parameter estimation was done off-line. A multilevel input signal was used for excitation. This signal gave good convergence for the NRPE method for all degrees of freedom. The maximum control force/momentum was limited to $300 \mathrm{~N}$ for surge and sway and $300 \mathrm{Nm}$ for yaw. These large control efforts may explain some of the good convergence, even for sway. For vehicles such as ships, which are not designed to move in the y-direction with any velocity, the convergence in sway may be rather poor. This is well known from ship dynamics.

Figure 3 indicates that the values for the estimated parameters were

$$
\begin{aligned}
X_{u|w|} & =-0.86, & X_{t} & =3.52 \\
Y_{v|v|} & =-1.48, & Y_{t} & =2.68 \\
N_{r|r|} & =-2.17, & N_{t} & =5.12
\end{aligned}
$$

These values were used for the simulation of the control law together with $\lambda=3.0$ for the desired control bandwidth and $\eta=0.1$. The proposed control strategy was applied to a multi-axis coupled motion for the underwater vehicle. The 3 robust trajectory controllers gave very satisfactory results for all degrees of freedom, see Fig. 4. The tracking performance will be reduced if the off-diagonal elements in the mass matrix or the uncertainty bounds are increased. A good parameter identification strategy makes it possible to reduce the uncertainty bounds and improve the tracking performance. The boundary layer thickness and the sliding surface are illustrated in Fig. 5.
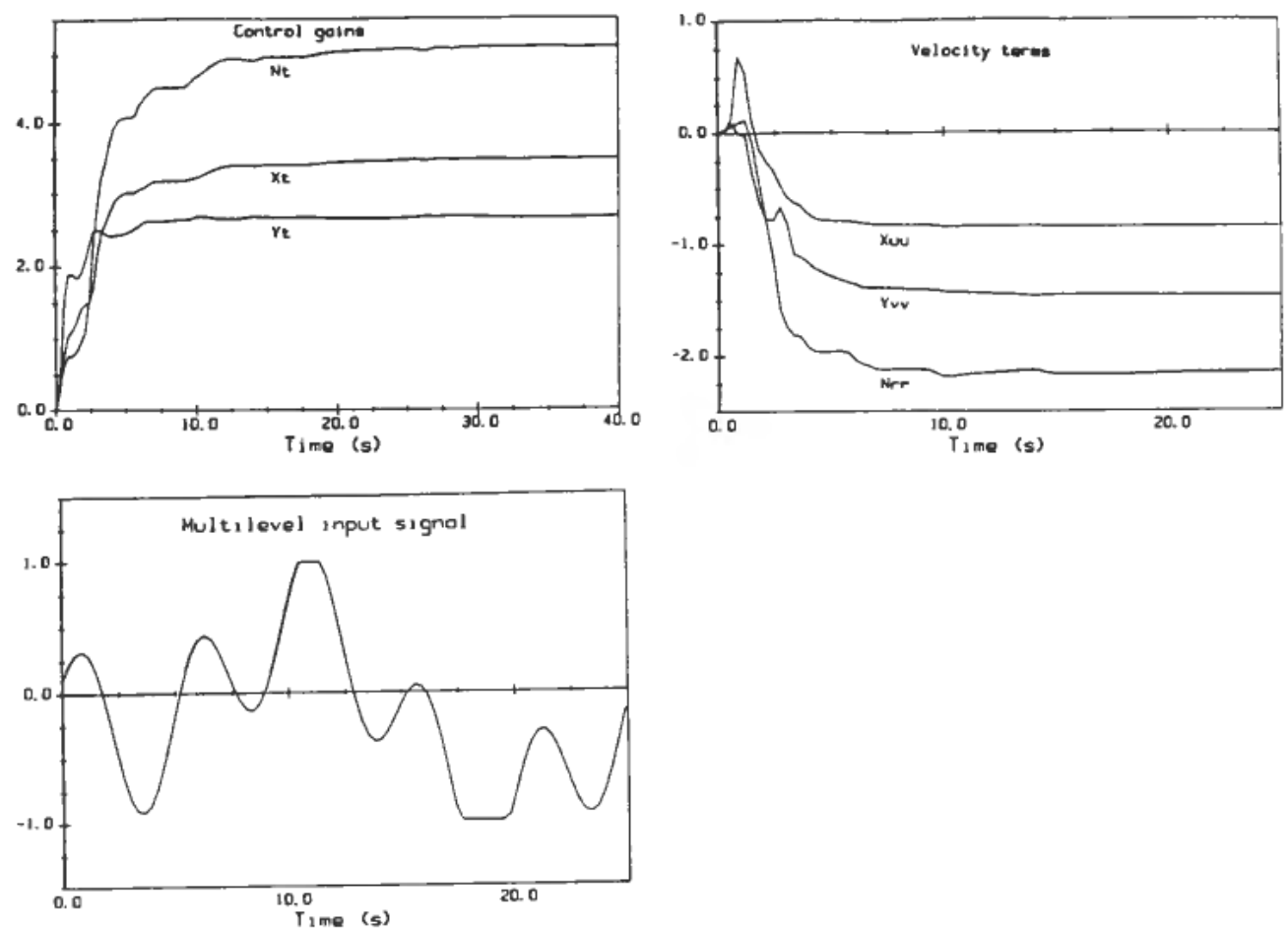

Figure 3. Estimated parameters from the non-linear recursive prediction error method and multilevel input signal. 

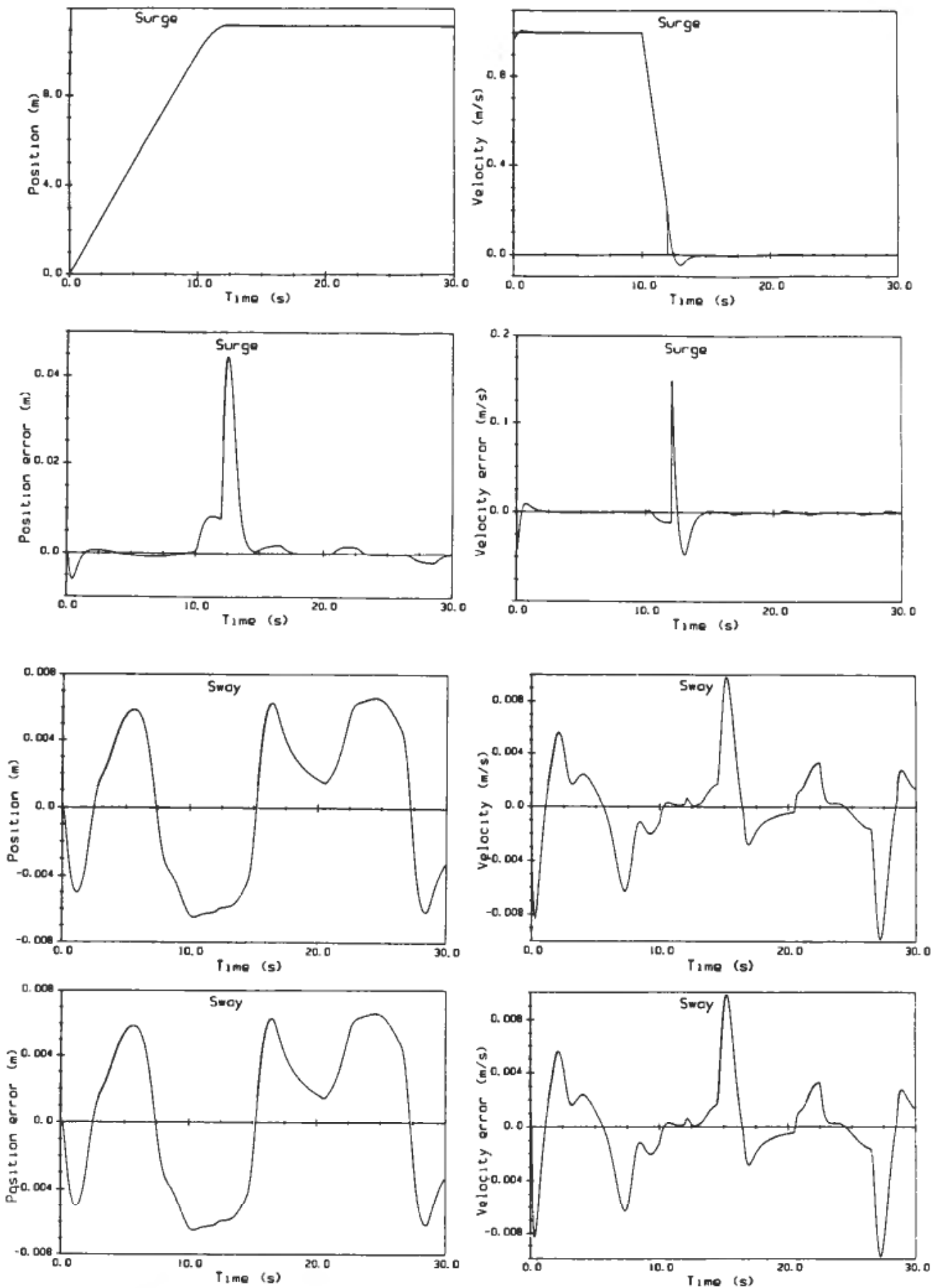

Figure 4. Actual position and velocity, position and velocity displacement errors and control efforts for surge, sway and yaw. 

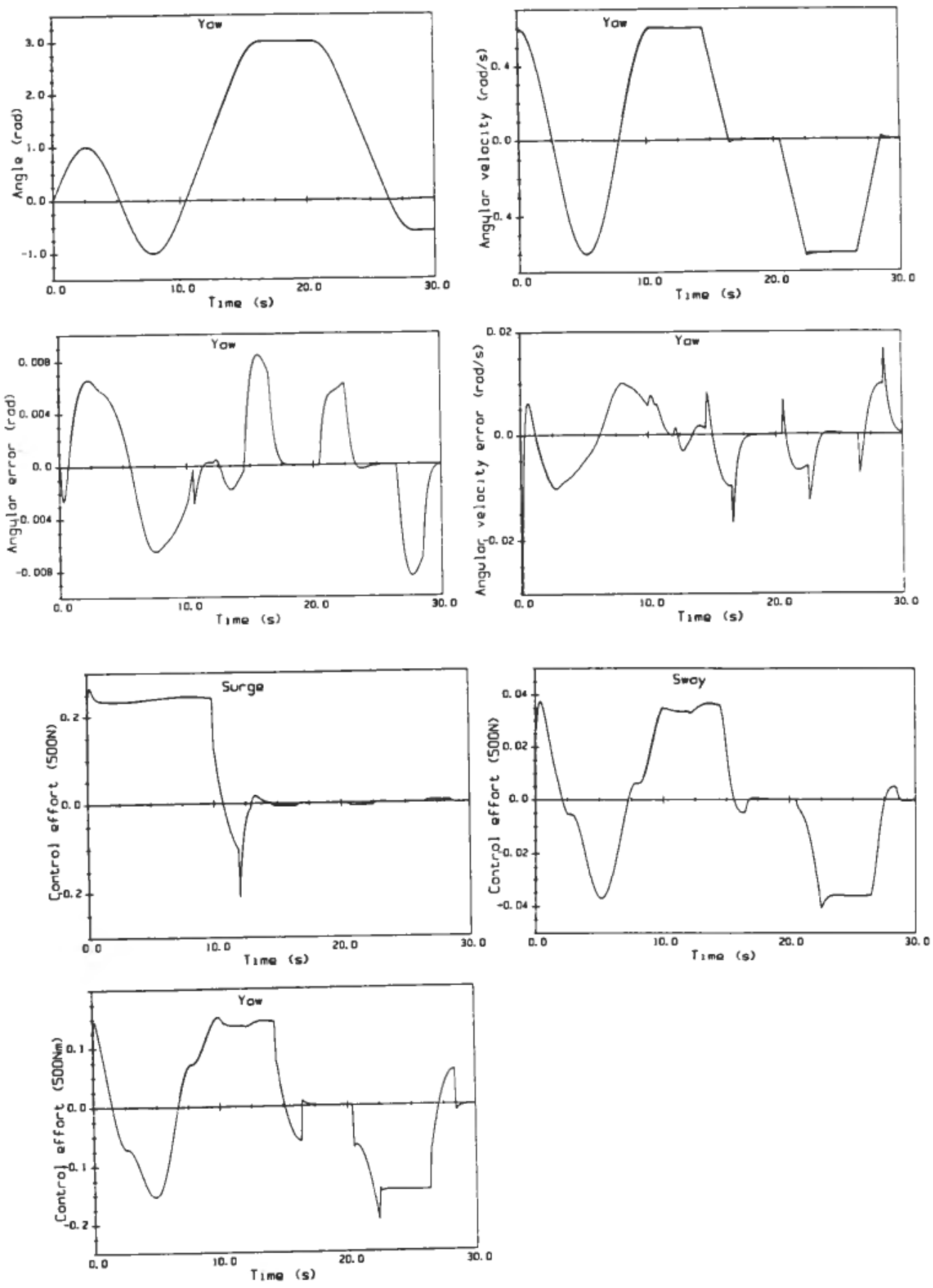

Figure 4. Actual position and velocity, position and velocity displacement errors and control efforts for surge, sway and yaw. 

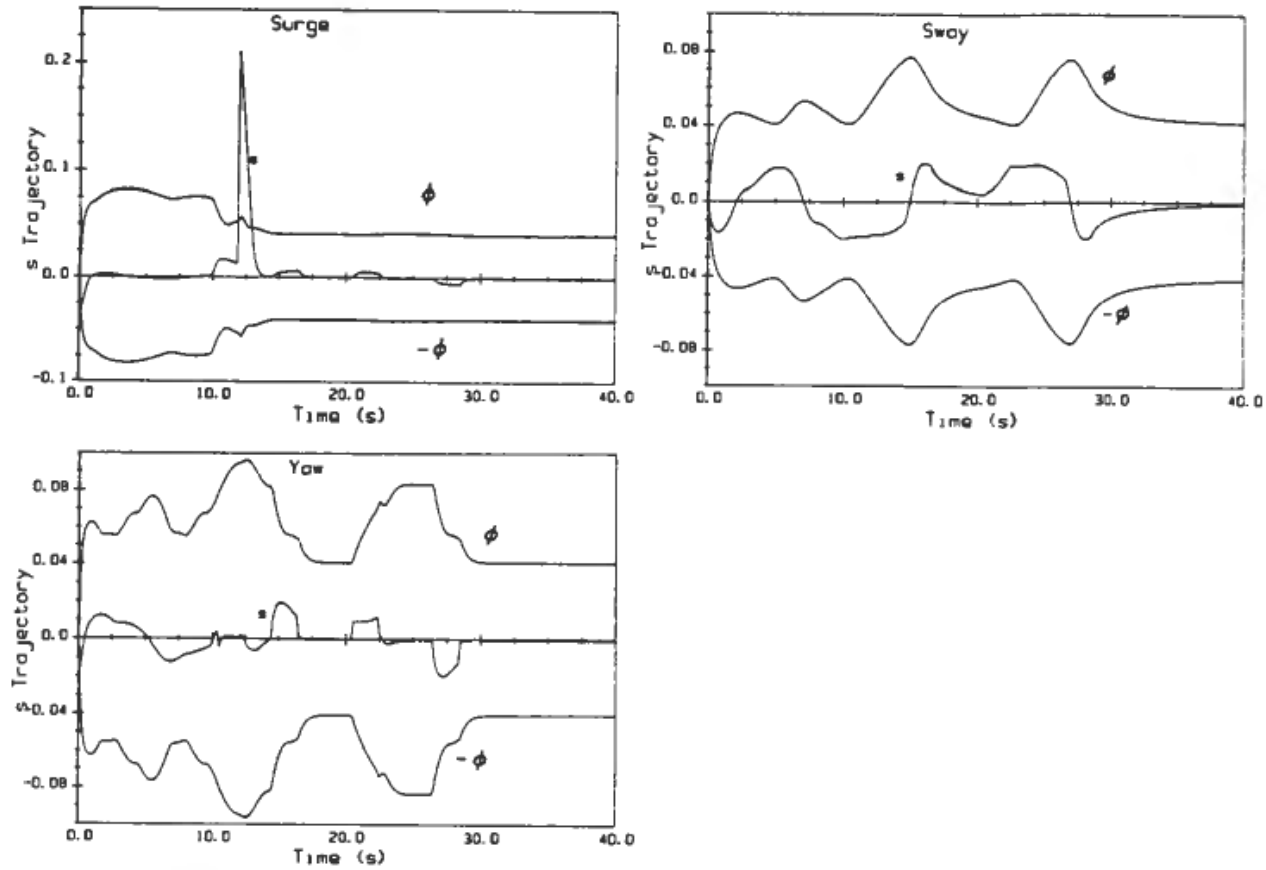

Figure 5. The boundary layer thickness and the sliding surface.

\section{Conclusion}

Non-linear control is especially necessary for an underwater vehicle that can move in several directions at the same time and has a large number of operating points. Underwater vehicles have complicated strongly coupled and highly nonlinear dynamics. Uncertainty in the added mass and velocity dependent terms reduce the performance and robustness. This paper has presented a non-linear selftuning control strategy based on a non-linear version of the recursive prediction error method combined with robust trajectory control. As this non-linear estimation algorithm decreases uncertainty in the model, this enables tighter control to be achieved, which leads to higher performance, see Fig. 4. Robust performance and stability is also improved especially as the uncertainty in the diagonal elements of the mass matrix are reduced (Egeland (1987)).

A flexible vehicle configuration is allowed through a self-tuning control scheme. Adding and removing different items of equipment such as television cameras and manipulator arms radically alter the vehicle's kinematic and hydrodynamic properties. Such unpredicted changes are easily dealt with by the self-tuning methodology. A self-tuning methodology also alleviates expensive model tests like the planar motion mechanism (PMM) test to determine the hydrodynamic coefficients. The vehicle's weight and equipment may be changed without requiring new model tests. It is also possible to extend the theory to a complete adaptive control law. A more complex model may also be used without producing too many problems with the identifiability.

\section{Acknowledgment}

This work has been sponsored by the Royal Norwegian Council for Scientific and Industrial Research through the Robotics Research Program at The Norwegian Institute of Technology. 


\section{REFERENCES}

Asada H. and Slotine, J. J. E. (1986). Robot Analysis and Control (John Wiley and Sons).

Delonga, D. M., Slotine, J. J. E. and Yoerger, D. R. (1987). Trajectory Control of Nonlinear, Time-Varying, Discrete-Time Systems using Discrete-Time Sliding Control, Fifth Int. Symp. on Unmanned Untethered Submersible Technology, Vol. 2, pp. 590-618, June 22-24, 1987.

EGELAND, O. (1987). On the robustness of the computed torque technique in manipulator control, Modeling Identification and Control, Vol. 8, 149-158.

Fossen, T. I. (1987). Control and Simulation of the Ballistic Properties of an Autonomous Underwater Vehicle, M.Sc. thesis, Div. Marine Systems Design, Norwegian Institute of Technology, Trondheim, in Norwegian.

LJUNG, L. (1987). System Identification, Theory for the User (Prentice Hall, 1987).

Newman J. N. (1977). Marine Hydrodynamics (MIT Press, Cambridge, Massachusetts).

SNAME, (1952). Nomenclature of treating the motion of a submerged body through a fluid, Technical and Research Bulletin, No. 1-5 (Society of Naval Architects and Marine Engineers, New York).

WENDEL, K. (1956). Hydrodynamic Masses and Hydrodynamic Moments of Inertia, TMB Translation 260, July 1956.

YoERGER, D. R. and SLOTINE, J. J. E. (1985). Robust trajectory control of underwater vehicles, IEEE Journal of Oceanic Engineering, 10, No. 4, pp. 462-470

ZHou, W. W. (1987). Identification of nonlinear marine systems, Ph.D thesis, Technical University of Denmark, June 1987. 